\title{
A note on crossing-trajectory effects in gas-particle turbulent flows
}

\author{
B. Oesterlé \\ LEMTA, Nancy-University, CNRS, ESSTIN, France.
}

\begin{abstract}
In the frame of Lagrangian stochastic dispersion models used to predict the behaviour of small inertial particles moving in a turbulent flow, crossing-trajectory effects are generally accounted for by modifying the integral time scales according to the famous analysis of Csanady (J. Atmos. Sci., 20, pp. 201-208, 1963). Here, an alternative theoretical analysis of the time correlation of the fluid velocity fluctuations along a particle trajectory is presented. Analytical expressions of the integral time scales of the fluid seen by the particles in isotropic turbulence are first derived in the asymptotic limit where the mean relative velocity is much larger than the particle velocity fluctuations, then a correction is proposed to extend their applicability over the whole range of mean relative velocity. These expressions do not depend on the presumed shape of the two-point fluid velocity correlations. The predicted time scales in the transverse direction differ from some available proposals in the literature, but are in agreement with other analyses based on an assumed functional form of the turbulence spectrum, at least in the limit of large mean relative velocity. Additionally, some comparisons with numerical predictions obtained in a synthetic Gaussian turbulence show that the present theoretical results are in good agreement with the computations in a large range of mean relative velocity and particle inertia.

Keywords: gas-particle flow, particle dispersion, crossing-trajectory, turbulence.
\end{abstract}

\section{Introduction}

As is well known, the behaviour of small inertial particles moving in a turbulent flow can be described by means of Lagrangian stochastic models that consist in building a proper stochastic process to predict the instantaneous velocity of the fluid seen by a discrete particle. The so-called crossing-trajectory effect is observed when the fluid and particle mean velocities differ due to some external force 
field, leading to significant decorrelation of the fluid velocity fluctuations along the discrete particle path. Due to continuity requirements, the decorrelation effect is larger in the directions perpendicular to the mean relative velocity. Crossingtrajectory effects are generally accounted for by modifying the integral time scales according to the famous analysis of Csanady [1]. Alternate formulations to express the non isotropic decrease of the correlation time scales were derived by Wang and Stock [2], Mei et al [3] and Derevich [4].

Here, we present a theoretical analysis of the time correlation of the fluid velocity fluctuations along a particle trajectory, under the assumption of isotropic turbulence which was also made in the above cited papers. Expressions of the integral time scales of the fluid seen are derived in the asymptotic limit where the mean relative velocity is much larger than the particle velocity fluctuations. A correction is then proposed to make these expressions valid in the whole range of mean relative velocity, taking the inertia effect into consideration. The results are compared with the various available expressions in [1-4] and with numerical predictions achieved through particle trajectory computations in a synthetic Gaussian turbulence as well.

\section{Analysis}

The autocovariance tensor of the fluid velocity fluctuations along the discrete particle path (fluctuations of the fluid seen) is defined by

$$
Q_{i j}^{*}(\mathbf{x}, t ; \tau)=\left\langle u_{i}^{\prime *}(t) u_{j}^{\prime *}(t+\tau)\right\rangle
$$

where $\mathbf{u}^{*}(t)=\mathbf{u}_{f}\left(\mathbf{x}_{p}(t), t\right)$ is the instantaneous velocity of the fluid at the particle location $\mathbf{x}_{p}(t)$, the prime denoting the fluctuating part of the velocity, and $\mathbf{x}=\mathbf{x}_{p}(t)$. Under the assumption of homogeneous stationary turbulence, where there is no dependence on position or time, we will use the shortened notation $Q_{i j}^{*}(\tau)=\left\langle u_{i}^{\prime *}(t) u_{j}^{\prime *}(t+\tau)\right\rangle \forall \mathbf{x}, t$.

As pointed out by Derevich [4,5], $Q_{i j}^{*}$ can be expressed in terms of the Eulerian two-point two-time velocity covariance tensor of the fluid measured in a reference frame moving with the mean fluid velocity,

$$
Q_{i j}(\mathbf{r}, \tau)=\left\langle u_{i}^{\prime}(\mathbf{x}, t) u_{j}^{\prime}(\mathbf{x}+\mathbf{r}, t+\tau)\right\rangle,
$$

and of the particle transition probability $\Psi(\mathbf{r}, \tau)$, which is the probability density function (PDF) of a particle having a displacement $\mathbf{r}$ during time $\tau$. The autocovariances of the fluid seen can be written as

$$
Q_{i j}^{*}(\tau)=\int Q_{i j}(\mathbf{r}, \tau) \Psi(\mathbf{r}, \tau) d \mathbf{r},
$$

where it is assumed that the particle displacement PDF is independent of the instantaneous velocity fluctuation of the fluid phase (independence approximation [6]), which means in particular that possible effects of preferential concentration are not considered here. 
In what follows, we are mainly interested by the integral time scales of the fluid seen by the particles in isotropic turbulence, under the effect of a constant mean relative velocity $\mathbf{V}$ between the fluid and the particles due to some external force field (equivalently, $\mathbf{V}$ is the mean particle velocity in a reference frame moving with the mean fluid velocity). Provided one axis of the co-ordinate system is aligned with this mean relative velocity, the study can be restricted to the diagonal components of the integral time scale matrix, defined by (no summation over Greek indices)

$$
T_{\alpha \alpha}^{*}=\left\langle u^{\prime 2}\right\rangle^{-1} \int_{0}^{\infty} Q_{\alpha \alpha}^{*}(\tau) d \tau,
$$

where it is assumed that there is no bias between the velocity variance of the fluid seen and the Eulerian one $\left\langle u^{\prime 2}\right\rangle$ (the subscript is omitted according to the assumed isotropy of the fluid turbulence). Without loss of generality, we will set $\mathbf{V}=V \mathbf{e}_{1}$.

\subsection{Approximate expressions of the space-time correlations}

In order to take advantage of eqn. (3), we have first to assess the spacetime correlations by means of some expressions which fulfill the asymptotic requirements for $\mathbf{r} \longrightarrow \mathbf{0}$ and $\tau \longrightarrow 0$. To this purpose, we know that Csanady [1] suggested that the lines of constant space-time covariance for longitudinal separation $r$ and time lag $\tau$ are ellipses obeying the equation

$$
\frac{r^{2}}{L_{f}^{2}}+\frac{\tau^{2}}{T_{E}^{2}}=\text { constant }
$$

where $L_{f}, T_{E}$ are the longitudinal integral length scale and the Eulerian integral time scale, respectively, assuming isotropic turbulence. In slight contrast with Csanady's formulation, here we use $T_{E}$ for sake of consistency with the limit $r \longrightarrow 0$, keeping in mind that $T_{E}$ is the Eulerian time scale measured in a reference frame moving with the mean fluid velocity ("moving Eulerian time scale"). Denoting the longitudinal two-point two-time correlation by $f(r, \tau)$, i.e.

$$
f(r, \tau)=\left\langle u^{\prime 2}\right\rangle^{-1} Q_{\alpha \alpha}\left(r \mathbf{e}_{\alpha}, \tau\right) \quad \forall \alpha
$$

(no summation over $\alpha$ ), Csanady's hypothesis comes down to assume that $f(r, \tau)$ is a function of the only variable

$$
\xi(r, \tau)=\left(\frac{r^{2}}{L_{f}^{2}}+\frac{\tau^{2}}{T_{E}^{2}}\right)^{1 / 2}
$$

that is

$$
f(r, \tau)=\varphi(\xi) .
$$

From eqns. (7)-(8) and from the definition of the integral length and time scales, namely $L_{f}=\int_{0}^{\infty} f(r, 0) d r$ and $T_{E}=\int_{0}^{\infty} f(0, \tau) d \tau$, it may be noticed that the 
function $\varphi(\xi)$ obeys

$$
\int_{0}^{\infty} \varphi(\xi) d \xi=1
$$

a property that will be used later.

To assess the two-point two-time covariances $Q_{\alpha \alpha}(\mathbf{r}, \tau)$ for any direction of the separation vector, we make use of the relationship between the longitudinal and transverse correlation functions $f(r, \tau)$ and $g(r, \tau)$ in isotropic turbulence,

$$
g(r, \tau)=f(r, \tau)+\frac{r}{2} \frac{\partial f}{\partial r}(r, \tau),
$$

to express the three diagonal components of the space-time covariance tensor as follows :

$$
\begin{aligned}
Q_{\alpha \alpha}(\mathbf{r}, \tau) & =\left\langle u^{\prime 2}\right\rangle\left((f(r, \tau)-g(r, \tau)) \frac{r_{\alpha}^{2}}{r^{2}}+g(r, \tau)\right) \\
& =f(r, \tau)+\frac{r^{2}-r_{\alpha}^{2}}{2 r} \frac{\partial f}{\partial r}(r, \tau)
\end{aligned}
$$

where $r_{\alpha}$ denotes the component of the separation vector $\mathbf{r}$ in direction $\alpha$, and $r^{2}=\sum_{\alpha=1}^{\alpha=3} r_{\alpha}^{2}=r_{i} r_{i}$. According to Csanady's hypothesis, $f(r, \tau)$ is assumed to obey eqn. (8), thus

$$
\frac{\partial f}{\partial r}(r, \tau)=\frac{\partial \xi}{\partial r} \varphi^{\prime}(\xi)=\frac{r}{L_{f}^{2} \xi} \varphi^{\prime}(\xi) .
$$

From eqns. (11)-(12), the diagonal space-time covariances for any direction of the separation vector can finally be written

$$
Q_{\alpha \alpha}(\mathbf{r}, \tau)=\left\langle u^{\prime 2}\right\rangle\left(\varphi(\xi)+\frac{r^{2}-r_{\alpha}^{2}}{2 L_{f}^{2} \xi} \varphi^{\prime}(\xi)\right)
$$

\subsection{Particle transition probability and the asymptotic case $V \gg\left\langle u^{\prime 2}\right\rangle^{1 / 2}$}

The main difficulty in using eqn. (3) on taking particle velocity fluctuations into account lies in the dependence of the particle displacement PDF upon the covariances $Q_{i j}^{*}(\tau)$, thus making the problem highly non linear. The analytical results arising from such approaches, due to Mei et al [3] and later by Derevich [4], can be summarized by the following expressions of the covariances of the fluid seen in the directions parallel $\left(Q_{11}^{*}\right)$ and perpendicular $\left(Q_{22}^{*}\right)$ to the mean relative velocity :

$$
\begin{aligned}
& Q_{11}^{*}(\tau)=\left\langle u^{\prime 2}\right\rangle \mu_{1}^{-1 / 2} \mu_{2}^{2} \exp \left[-\frac{\pi}{4} \frac{\tau^{2}}{T_{E}^{2}}\left(1+\frac{\gamma^{2}}{\mu_{1}}\right)\right], \\
& Q_{22}^{*}(\tau)=\frac{1}{2} Q_{11}^{*}\left[1+\frac{\mu_{2}}{\mu_{1}}\left(1-\frac{\pi}{2} \frac{\tau^{2}}{T_{E}^{2}} \frac{\gamma^{2}}{\mu_{1}}\right)\right],
\end{aligned}
$$


where

$$
\gamma=\frac{V T_{E}}{L_{f}} \quad \text { and } \quad \mu_{\alpha}=1+\frac{\sigma_{\alpha}^{2}(\tau)}{4} .
$$

Unfortunately, eqns. (14)-(15) are clearly unclosed because their right hand sides involve the r.m.s. particle displacement $\sigma_{\alpha}$ which depends on $Q_{\alpha \alpha}^{*}$ as mentioned just above. Therefore the only means of determining the integral time scales of the fluid seen from such expressions would be through some numerical iterative procedure. As our objective is to find the integral time scales $T_{11}^{*}$ and $T_{22}^{*}$ under the form of analytical expressions that can be introduced in stochastic dispersion models, we will first investigate the simple asymptotic limit where the mean relative velocity is much larger than the particle velocity fluctuations, i.e. $V \gg\left\langle u^{\prime 2}\right\rangle^{1 / 2}$ (or $\gamma \gg 1$ ) and then we will try to extend the obtained expressions in order that they remain valid in the opposite limit where only the inertia effect is present.

In the case $\gamma \gg 1$ the r.m.s. particle displacement can be neglected compared to the mean particle displacement during time $\tau$ which is $\langle\mathbf{r}\rangle=\mathbf{V} \tau$. This assumption leads to a drastic simplification of the particle displacement PDF, which can be expressed as

$$
\Psi(\mathbf{r}, \tau)=\delta(\mathbf{r}-\mathbf{V} \tau),
$$

and so the corresponding covariance of the fluid seen, hereafter denoted by $\widetilde{Q}_{\alpha \alpha}^{*}(\tau)$, obeys

$$
\widetilde{Q}_{\alpha \alpha}^{*}(\tau)=\int Q_{\alpha \alpha}(\mathbf{r}, \tau) \delta(\mathbf{r}-\mathbf{V} \tau) d \mathbf{r}=Q_{\alpha \alpha}(\mathbf{V} \tau, \tau) .
$$

Using eqn. (13) to express $Q_{\alpha \alpha}(\mathbf{V} \tau, \tau)$, the expressions of the time scales $\widetilde{T}_{\alpha \alpha}^{*}$ can be obtained by integration (the tilde stands for the case $\gamma \gg 1$ ). In the direction parallel to the mean relative velocity (direction 1), eqns. (13), (18)and (9) yield

$$
\widetilde{T}_{11}^{*}=\int_{0}^{\infty} \varphi(\xi) d \tau=\frac{T_{E}}{\sqrt{1+\gamma^{2}}} \int_{0}^{\infty} \varphi(\xi) d \xi=\frac{T_{E}}{\sqrt{1+\gamma^{2}}}
$$

where we used

$$
\xi=\sqrt{\frac{V^{2} \tau^{2}}{L_{f}^{2}}+\frac{\tau^{2}}{T_{E}^{2}}}=\frac{\tau}{T_{E}} \sqrt{1+\gamma^{2}} .
$$

In the directions perpendicular to $\mathbf{V}$ (for example $\alpha=2$ ), the continuity effect is taken into account by using eqn. (13) with $r=r_{1}=V \tau, r_{2}=0$ :

$$
Q_{22}(\mathbf{V} \tau, \tau)=\left\langle u^{\prime 2}\right\rangle\left[\varphi(\xi)+\frac{V^{2} \tau^{2}}{2 L_{f}^{2} \xi} \varphi^{\prime}(\xi)\right]=\left\langle u^{\prime 2}\right\rangle\left[\varphi(\xi)+\frac{\gamma^{2} \xi \varphi^{\prime}(\xi)}{2\left(1+\gamma^{2}\right)}\right]
$$

hence, after integration by parts :

$$
\widetilde{T}_{22}^{*}=\frac{T_{E}}{\sqrt{1+\gamma^{2}}}\left[1+\frac{\gamma^{2}}{2\left(1+\gamma^{2}\right)} \int_{0}^{\infty} \xi \varphi^{\prime}(\xi) d \xi\right]=\frac{T_{E}}{\sqrt{1+\gamma^{2}}}\left[1-\frac{\gamma^{2}}{2\left(1+\gamma^{2}\right)}\right] .
$$


It is worth noticing that the derivation of expressions (19) and (21) does not require to prescribe the shape of the double velocity correlations of the fluid, except that Csanady's assumption implies that the longitudinal two-point one-time correlation and the one-point two-time correlation have similar shapes.

\subsection{Extension to smaller mean relative velocity}

Let us introduce the integral time scale of the fluid seen for $V=0$, denoted by $T_{0}^{*}$, which depends on the Stokes number $S t_{E}=\tau_{P} / T_{E}$ where $\tau_{P}$ is the particle relaxation time. As is well known, for tracer particles $\left(S t_{E} \ll 1\right) T_{0}^{*}$ is equal to the Lagrangian integral time scale $T_{L}$, whereas for high inertia particles, such that $S t_{E} \gg 1, T_{0}^{*}$ tends to the moving Eulerian time scale $T_{E}\left(>T_{L}\right):$ this is the socalled inertia effect [7]. The time scale $T_{0}^{*}$ can be estimated in terms of the Stokes number from the semi-empirical correlation proposed by Wang and Stock [2] or from the analytical formula derived by Derevich [5], for example.

The main objective here is to introduce the Stokes number dependence into the evaluation of the integral time scales when crossing trajectory effects are present. Any extension of the analytical expressions of $\widetilde{T}_{\alpha \alpha}^{*}$ obtained at large mean relative velocity must fulfill the condition that $T_{\alpha \alpha}^{*} \rightarrow T_{0}^{*}$ for $\gamma \rightarrow 0$. In order to meet this requirement, the simple method suggested here is to replace $T_{E}$ by $T_{0}^{*}$ in the results derived for large $\gamma$, i.e. in eqns. (19)-(21). Therefore the proposed formulae are

$$
\begin{aligned}
& T_{11}^{*}=\frac{T_{0}^{*}}{\sqrt{1+\gamma^{* 2}}}, \\
& T_{22}^{*}=\frac{T_{0}^{*}}{\sqrt{1+\gamma^{* 2}}}\left[1-\frac{\gamma^{* 2}}{2\left(1+\gamma^{* 2}\right)}\right],
\end{aligned}
$$

where

$$
\gamma^{*}=\frac{V T_{0}^{*}}{L_{f}} .
$$

\section{Discussion}

Our expressions of the integral time scales of the fluid seen in the case $\gamma \gg 1$, eqns. (19) and (21), are in agreement with the results of Mei et al. [3] and Derevich [4], as can be proved by integrating eqns. (14)-(15) with $\mu_{1} \rightarrow 1, \mu_{2} \rightarrow 1$ (according to the hypothesis $V \gg\left\langle u^{\prime 2}\right\rangle^{1 / 2}$ ). Let us emphasize, however, that no assumption has been made here concerning the velocity correlation functions or the spectrum tensor of the fluid, whereas Mei et al. [3] and Derevich [4] had to prescribe a functional form of the spectrum tensor (namely the Kraichnan's model spectrum). Unfortunately, no comparison can be made with these works in the general case where the velocity fluctuations are not small compared to $V$, due to the already mentioned unclosedness of the correlation expressions (14)-(15). 
Further comparison can be made with the proposals by Csanady [1] and by Wang and Stock [2]. Csanady [1] assumed exponential forms of the longitudinal two-point correlation $f(r, 0)$ and of the one-point Eulerian time correlation $f(0, \tau)$, and considered that the moving Eulerian time scale $T_{E}$ equals the Lagrangian time scale $T_{L}$. Therefore the integral time scale of the fluid seen by a heavy particle for $V=0$ is equal to the fluid Lagrangian time scale $T_{L}$ whatever the particle inertia, and the formula derived by Csanady in the asymptotic case $\gamma \gg 1$ remains valid in the opposite case of very small relative velocity. Therefore the above expression (22) of the integral time scale of the fluid seen in the direction of the mean relative velocity is in line with Csanady's analysis.

For the particle dispersion in the lateral directions, Csanady suggested an empirical formula so as to obtain the correct asymptotic behaviour for $V \longrightarrow \infty$, i.e. $\widetilde{T}_{22}^{*}=\widetilde{T}_{11}^{*} / 2$. Extending his proposal using $T_{0}^{*}$ as explained in section 2.3 (keeping in mind that $T_{E}=T_{L}$ was assumed in Csanady's work), the Csanadylike formulation of the transverse integral time scales of the fluid seen would be :

$$
T_{22, C}^{*}=\frac{T_{0}^{*}}{\sqrt{1+4 \gamma^{* 2}}} .
$$

Later, Wang and Stock [2] developed a theoretical analysis including the effect of particle inertia. Still using exponential functions for $f(r, 0)$ and $f(0, \tau)$, they

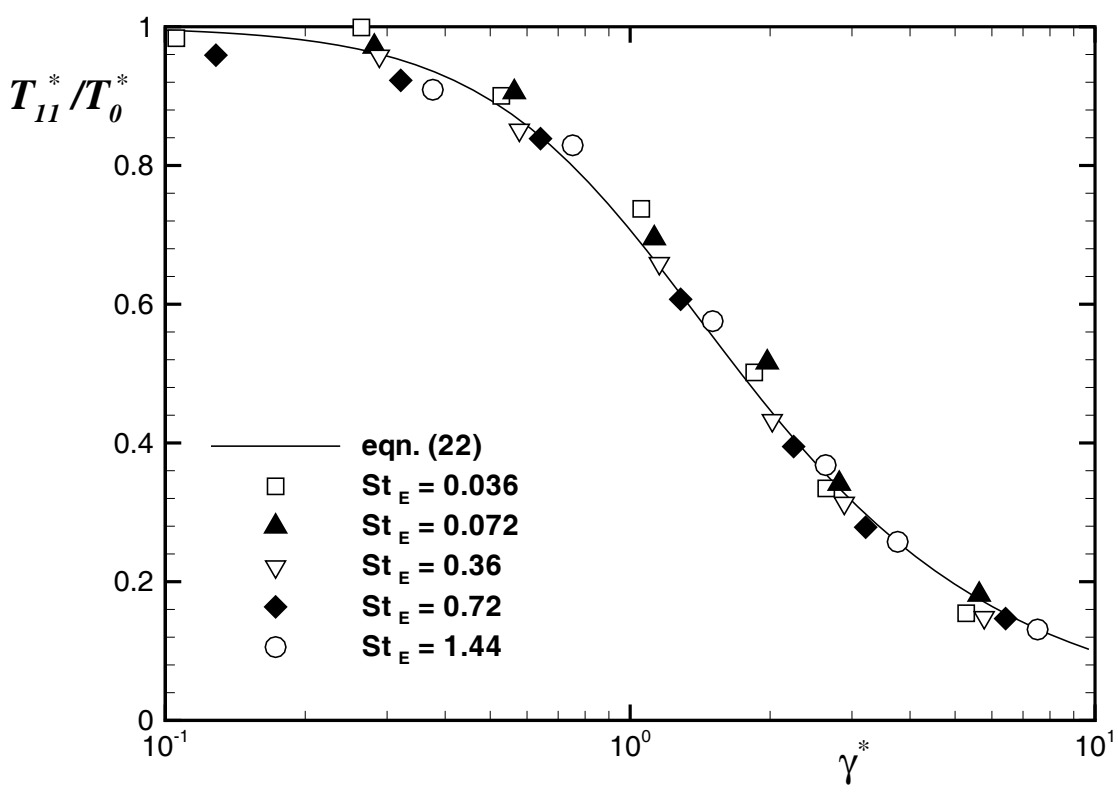

Figure 1: Integral time scale ratio $T_{11}^{*} / T_{0}^{*}$ (direction of the mean relative velocity) as a function of the parameter $\gamma^{*}$. 


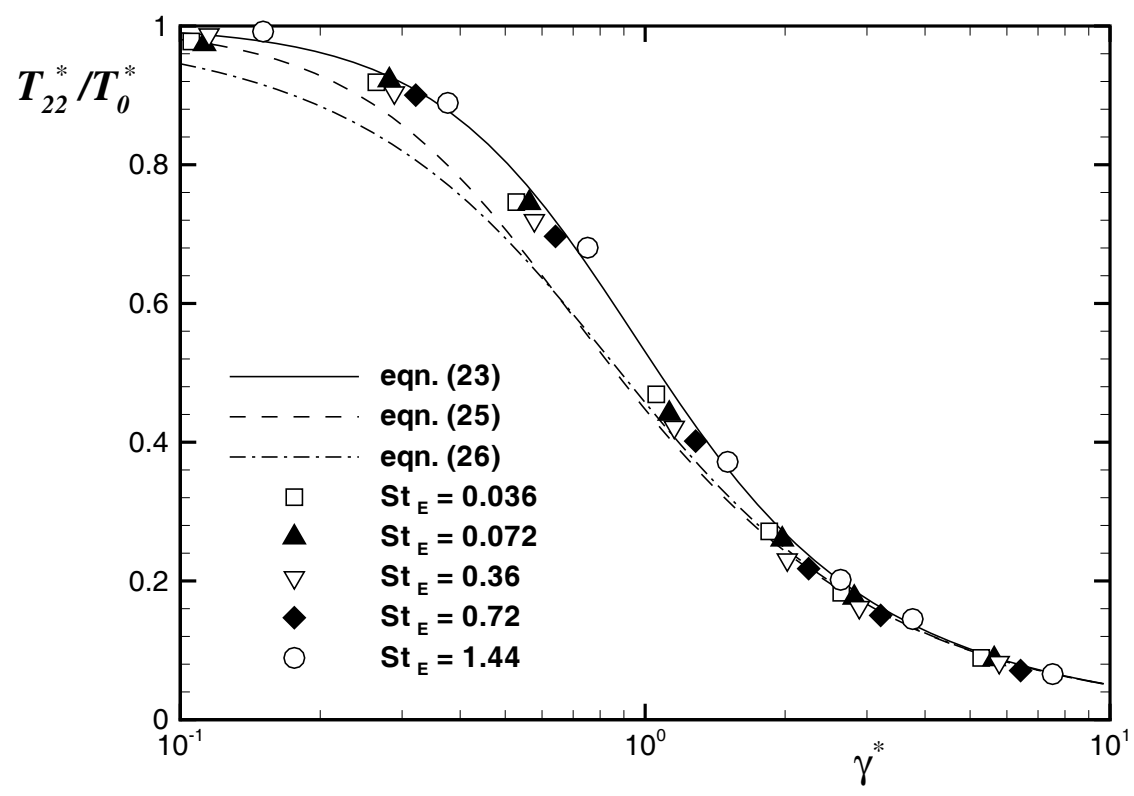

Figure 2: Integral time scale ratio $T_{22}^{*} / T_{0}^{*}$ (direction perpendicular to the mean relative velocity) as a function of the parameter $\gamma^{*}$.

extended the idea of Csanady in assuming that the combined influence of $\tau, T_{0}^{*}$ and $\gamma^{*}$ in the correlation of the fluid seen $Q_{11}^{*}$ can only appear through the variable $\left(\tau / T_{0}^{*}\right) \sqrt{1+\gamma^{* 2}}$. Whereas their result is the same as ours in the direction of $\mathbf{V}$, the following expression was obtained in the transverse directions :

$$
T_{22, W S}^{*}=\frac{T_{0}^{*}}{\sqrt{1+\gamma^{* 2}}}\left[1-\frac{\gamma^{*}}{2 \sqrt{1+\gamma^{* 2}}}\right] .
$$

To sum up, regarding the decorrelation of the fluid seen in the direction of the mean relative velocity it is found that there is no difference between our result and the available proposals in the literature. In contrast, some discrepancies can be found in the expressions of the time scale of the fluid seen in the transverse directions, see eqns. (23), (25) and (26). In Fig. 1 and 2, these various formulae are compared with some numerical predictions issued from particle trajectory computations in a synthetic Gaussian turbulence whose properties are as follows (see [8]) : Von Karman spectrum, semi-Gaussian Eulerian time correlation, $T_{E}=$ 1.39 and $T_{L}=0.44$ (arbitrary units), $R e_{L} \approx 180$ (turbulence Reynolds number based on integral length scale $L_{f}$ ). For each value of the Stokes number $S t_{E}, 10^{5}$ particles were tracked.

As can be observed in Fig. 1 which displays the time scale ratio $T_{11}^{*} / T_{0}^{*}$ (i.e. in the direction of $\mathbf{V}$ ) as a function of $\gamma^{*}$, the analytical prediction compares very 
well with the numerical simulation whatever the Stokes number. As regards the time scale of the fluid seen in the transverse directions, comparison of the three expressions (23), (25) and (26) is provided by Fig. 2, which shows slight but significant discrepancies between the analytical predictions. Equation (23) can be seen to lead to the best agreement with the numerical predictions.

\section{Conclusion}

The theoretical expressions derived here for the integral time scales of the fluid seen by the particles are in line with the predictions of Mei et al. [3] and Derevich [4] in the limit of large mean relative velocity. These expressions have been extended to make them consistent with the presence of inertia effect whatever the mean relative velocity may be. The main result is that the predicted time scales in the transverse direction differ from both proposals by Csanady [1] and by Wang and Stock [2]. Comparison of the various available expressions with some numerical predictions obtained in a synthetic Gaussian turbulence, in a large range of mean relative velocity and particle inertia, shows that the transverse decorrelation due to crossing-trajectory effect is better predicted by the expression arising from the present analysis.

\section{References}

[1] Csanady, G.T., Turbulent diffusion of heavy particles in the atmosphere. $J$ Atmos Sci, 20, pp. 201-208, 1963.

[2] Wang, L.P. \& Stock, D.E., Dispersion of heavy particles by turbulent motion. J Atmosph Sci, 50, pp. 1897-1913, 1993.

[3] Mei, R., Adrian, R.J. \& Hanratty, T.J., Particle dispersion in isotropic turbulence under Stokes drag and Basset force with gravitational settling. $J$ Fluid Mech, 225, pp. 481-495, 1991.

[4] Derevich, I.V., Influence of internal turbulent structure on intensity of velocity and temperature fluctuations of particles. Int J Heat \& Mass Transfer, 44, pp. 4505-4521, 2001.

[5] Derevich, I.V., Statistical modelling of mass transfer in turbulent two-phase dispersed flows - 1. Model development. Int J Heat \& Mass Transfer, 43, pp. 3709-3723, 2000.

[6] Weinstock, J., Lagrangian-Eulerian relation and the independence approximation. Phys Fluids, 19, pp. 1702-1711, 1976.

[7] Reeks, M., On the dispersion of small particles suspended in an isotropic turbulent field. J Fluid Mech, 83, pp. 529-546, 1977.

[8] Thomas, L. \& Oesterlé, B., An investigation of crossing trajectory effects in turbulent shear flows. Proc. ASME FED Summer Meeting, FEDSM'05, Houston, Texas, 2005. Paper No FEDSM2005-77303. 